\title{
Microscopic studies of multiphonon nuclear spectra.
}

\author{
N. Lo ludice ${ }^{\dagger},{ }^{a}$ F. Andreozzi, ${ }^{a}$ F. Knapp, ${ }^{b}$ A. Porrino ${ }^{a}$ and J. Kvasil ${ }^{b}$ \\ ${ }^{a}$ Dipartimento di Scienze Fisiche, Universitá di Napoli Federico II \\ and Istituto Nazionale di Fisica Nucleare, \\ Monte S. Angelo, via Cintia, I-80126 Napoli, Italy \\ ${ }^{b}$ Institute of Particle and Nuclear Physics, Charles University, \\ V Holešovičkách 2, CZ-18000 Praha 8, Czech Republic \\ E-mail: loiudice@na.infn.it, Andreozzi@na.infn.it, \\ knappeipnp.troja.mff.cuni.cz, \\ Porrino@na.infn.it,kvasileipnp.troja.mff.cuni.cz
}

\begin{abstract}
A microscopic approach to nuclear spectroscopy, especially deviced for treating multiphonon excitations, is outlined. It consists in constructing a set of equations of motion within a subspace spanned by states which are tensor products of a given number of microscopic phonons. These equations are solved iteratively, starting from the particle-hole vacuum, generating a basis of microscopic multiphonon states. In such a highly correlated basis, the Hamiltonian can be brought easily to diagonal form, with no approximations. The transition amplitudes are also computed exactly through simple recursion relations. Illustrative numerical applications are given.
\end{abstract}

Workshop on Photon Strength Functions and Related Topics

June 17-20 2007

Prague, Czech Republic

*Work partly supported by the Italian Ministero della Istruzione Universitá e Ricerca (MIUR), the Czech grant agency under the contract No. 202/06/0363) and the Czech Ministry of Education (contract No. VZ MSM 0021620859)

† Speaker. 


\section{Introduction}

The microscopic studies of collective motion in nuclei are usually performed in mean field theories like random-phase approximation (RPA) [1, 2]. RPA, however, accounts only for the fragmentation of the collective mode due to its decay to single-particle states (Landau damping) [3]. Important anharmonic features, like the collisional damping, responsible for the so called spreading width, are outside the domain of mean field theories. Moreover, the evidence of multiphonon spectra, with their inherent anharmonicities, has been growing rapidly in recent years. Low-lying double-quadrupole, double-octupole and mixed quadrupole-octupole multiplets have been detected in resonance fluorescence scattering experiments [4]. A class of multiphonon quadrupole excitations known as proton-neutron (F-spin) mixed symmetry states has been discovered in experiments combining different $\gamma$-ray spectroscopy techniques [5, 6, 7]. Quadrupole three-phonon excitations have been also observed $[6,7]$. At high energy, the double giant dipole resonance has been established in a number of different reactions $[8,9]$. In deformed nuclei, a recent $\gamma$ cascade experiment [10] could disentangle the $M 1$ from the $E \lambda$ deexcitations and provide evidence of a scissors mode $[11,12]$ built on excited states. A series of $(p, t)$ transfer reaction experiments have populated an impressive number of $0^{+}$states up to $3 \mathrm{MeV}[13,14,15,16]$.

In order to properly describe the fragmentation of the one-phonon collective modes as well as the anharmonic features of the multiphonon spectra, it is compulsory to go beyond mean-field theories. To this purpose, Boson expansion techniques, to be implemented under some Fermion-Boson mapping prescription, have been developed long ago [17, 18, 19]. A celebrated application of this technique is represented by the phenomenological interacting-boson model (IBM), so successful in providing systematics of the low-energy multiphonon spectra [20]. The IBM, indeed, can be considered a phenomenological realization of a Marumori Fermion-Boson mapping [21].

On the other hand, realistic microscopic calculations exploiting explicitly the Boson expansion techniques are not very many, because of the slow convergence of the Boson series. The most widely adopted approaches are only inspired by the Fermion-Boson mapping. Notable examples are the nuclear field theory [22, 23], especially suitable for characterizing the anharmonicities of the vibrational spectra and the spreading widths of the giant resonances, and the quasiparticle-phonon model (QPM) [24], extensively adopted to describe multiphonon excitations and their anharmonic features [25, 26, 27]. It is worth mentioning also some microscopic multiphonon approaches based on the iterative solution of equations of motion [28, 29].

Moving along similar lines, we have developed a new equation of motion method [30] which generates iteratively a basis of multiphonon states, built out of phonons constructed in the TammDancoff approximation (TDA) [1]. The basis is then used to solve exactly the nuclear eigenvalue problem in the space spanned by such a correlated basis. The method holds for the most general Hamiltonian.

We first outline briefly the mean field approaches and the limits of their validity. We then illustrate the main steps of our multiphonon method and show how the procedure is implemented, with no approximations, in the specific case of ${ }^{16} \mathrm{O}$, which, because of its highly complex shell structure, represents a severe test for any microscopic calculation. 


\section{Mean field approaches}

In purely collective models, one constructs a harmonic oscillator (HO) Hamiltonian in the collective coordinate $\alpha_{\lambda}$, of multipolarity $\lambda$, and its conjugate momentum $\pi_{\lambda}$, which fulfills the eigenvalue equations

$$
\left[H, O_{\lambda}^{\dagger}\right]=\hbar \omega_{\lambda} O_{\lambda}^{\dagger},
$$

where $O_{\lambda}^{\dagger}$ and $O_{\lambda}$ are, respectively, the quanta creation and annihilation operators with respect to the bosonic vacuum $\mid 0>$. The collective mode is described by the first excited state $\left|\lambda>=O_{\lambda}^{\dagger}\right| 0>$, of energy $\omega_{\lambda}$. Such a state collects the whole strength of the coordinate $\alpha_{\lambda}$.

The nuclear Hamiltonian $H$ is far from being harmonic. Its form in second quantization is

$$
H=\sum_{i} \varepsilon_{i} a_{i}^{\dagger} a_{i}+\frac{1}{4} \sum_{i j k l} V_{i j k l} a_{i}^{\dagger} a_{j}^{\dagger} a_{l} a_{k}
$$

where $\varepsilon_{i}$ are the single-particle energies, $V_{i j k l}$ the antisymmetrized matrix elements of the nucleonnucleon interaction, $a_{i}^{\dagger}\left(a_{i}\right)$ the creation (annihilation) particle operators with respect to the Fermionic vacuum.

It is nonetheless possible to turn formally the nuclear eigenvalue problem into a HO-like eigenvalue equation [1,2]. If $|0\rangle$ is the lowest eigenstate of $H$, namely the nuclear ground state, one can always define operators $O_{\lambda}$ and $O_{\lambda}^{\dagger}$ satisfying the conditions

$$
O_{\lambda}|0\rangle=0, \quad\left|\lambda>=O_{\lambda}^{\dagger}\right| 0>
$$

for any eigenstate $\mid \lambda>$ of $\mathrm{H}$. Under the above constraints, the operators $O_{\lambda}$ and $O_{\lambda}^{\dagger}$ satisfy the following HO-like equations

$$
\left[H, O_{\lambda}^{\dagger}\right]|0\rangle=\omega_{\lambda} O_{\lambda}^{\dagger}|0\rangle=\left(E_{\lambda}-E_{0}\right) O_{\lambda}^{\dagger}|0\rangle .
$$

RPA consists in solving the above eigenvalue equations in a restricted space spanned by particlehole $(p h)$ states, assuming that $\mid 0>$ is the true, highly correlated, ground state. Under this assumption, the Boson-like operators takes the form

$$
0_{\lambda}^{\dagger}=\sum_{p h}\left(Y_{p h}^{\lambda} a_{p}^{\dagger} a_{h}-Z_{p h}^{\lambda} a_{h}^{\dagger} a_{p}\right) .
$$

The explicit eigenvalue equations are obtained by expanding the commutator in Eq.(2.4) and making use of the approximation

$$
\begin{aligned}
& <0\left|a_{p}^{\dagger} a_{p^{\prime}}\right| 0>\sim<\left|a_{p}^{\dagger} a_{p^{\prime}}\right|>=0 \\
& <0\left|a_{h}^{\dagger} a_{h^{\prime}}\right| 0>\sim<\left|a_{h}^{\dagger} a_{h^{\prime}}\right|>=\delta_{h h^{\prime}},
\end{aligned}
$$

where |\rangle is the Hartree-Fock $p h$ vacuum. The replacement of the correlated vacuum $\mid 0>$ with the unperturbed one $\mid>$ represents the quasi boson approximation (QBA). One finally obtains

$$
\left|\begin{array}{ll}
A & B \\
B^{*} & A^{*}
\end{array}\right|\left|\begin{array}{l}
Y_{\lambda} \\
Z_{\lambda}
\end{array}\right|=h \omega_{\lambda}\left|\begin{array}{l}
Y_{\lambda} \\
-Z_{\lambda}
\end{array}\right|
$$


where

$$
\begin{aligned}
& A_{p h, p^{\prime} h^{\prime}}=\left(\varepsilon_{p}-\varepsilon_{h}\right) \delta_{p p^{\prime}} \delta_{h h^{\prime}}+V_{p h^{\prime} h^{\prime} p}, \\
& B_{p h, p^{\prime} h^{\prime}}=V_{p p^{\prime} h h^{\prime}} .
\end{aligned}
$$

The RPA states $|\lambda\rangle=O_{\lambda}^{\dagger}|0\rangle$ are normalized according to

$$
\left\langle\lambda \mid \lambda^{\prime}\right\rangle=\left\langle 0\left|0_{\lambda} 0_{\lambda^{\prime}}^{\dagger}\right| 0\right\rangle=\left\langle 0\left|\left[0_{\lambda}, 0_{\lambda^{\prime}}^{\dagger}\right]\right| 0\right\rangle \cong\left\langle\left|\left[0_{\lambda}, 0_{\lambda^{\prime}}^{\dagger}\right]\right|\right\rangle=\delta_{\lambda \lambda^{\prime}},
$$

where, once again, use of the QBA has been made. In virtue of this approximation, the normalization condition yields

$$
\sum_{p h}\left(Y_{p h}^{\lambda *} Y_{p h}^{\lambda^{\prime}}-Z_{p h}^{\lambda *} Z_{p h}^{\lambda^{\prime}}\right)=\delta_{\lambda \lambda^{\prime}}
$$

The transition amplitudes for the generic one-body operator $W$ are given by

$$
\langle\lambda|W| 0\rangle=\left\langle 0\left|\left[0_{\lambda}, W\right]\right| 0\right\rangle \cong\left\langle\left|\left[0_{\lambda}, W\right]\right|\right\rangle=\sum_{p h}\left(Y_{p h}^{\lambda *} W_{p h}+Z_{p h}^{\lambda *} W_{h p}\right) .
$$

For open shell nuclei, one has to move from a particle to a quasiparticle formalism by means of the canonical Bogoliubov transformation

$$
\begin{aligned}
& \alpha_{\alpha}^{\dagger}=u_{\alpha} a_{\alpha}^{\dagger}-v_{\alpha} a_{\bar{\alpha}}, \\
& \alpha_{\alpha}=u_{\alpha} a_{\alpha}-v_{\alpha} a_{\bar{\alpha}}^{\dagger}
\end{aligned}
$$

where $\bar{\alpha}$ denotes time reversal. The quasiparticle RPA (QRPA) states have the form

$$
|\lambda\rangle=0_{\lambda}^{\dagger}|0\rangle=\sum_{\alpha \beta}\left\{Y_{\alpha \beta}^{\lambda} \alpha_{\alpha}^{\dagger} \alpha_{\beta}^{\dagger}-Z_{\alpha \beta}^{\lambda} \alpha_{\beta} \alpha_{\alpha}\right\}|0\rangle
$$

and yield the QRPA transition amplitudes

$$
\langle\lambda|W| 0\rangle=\left\langle 0\left|\left[0_{\lambda}, W\right]\right| 0\right\rangle \simeq \sum_{\alpha>\beta}\left(Y_{\alpha \beta}^{\lambda^{*}} W_{\alpha \beta}+Z_{\alpha \beta}^{\lambda^{*}} W_{\beta \alpha}\right)\left(u_{\alpha} v_{\beta}+\tau v_{\alpha} u_{\beta}\right),
$$

where $\tau=+1$ or -1 according that the operator $W$ is even or odd under time-reversal.

Clearly, the RPA equations, to be solved numerically, have a much richer structure than the purely Bosonic one (2.1). Indeed, they yield not one but many eigenvalues. Only one state, however, is collective and gets a large fraction of the strength. The others are non collective and get a relatively small strength. Thus, the mode gets fragmented because of its decay to single-particle levels (Landau damping).

It is to be pointed out, once more, that RPA is not exact but relies on the QBA. This approximation can be removed by using from the beginning the Hartree-Fock $p h$ vacuum, $|0>=|>$. In such a case, one obtains the TDA eigenvalue equations

$$
\sum_{p^{\prime} h^{\prime}} A\left(p h ; p^{\prime} h^{\prime}\right) X^{\lambda}\left(p^{\prime} h^{\prime}\right)=\left(E_{\lambda}-E_{0}\right) X^{\lambda}(p h)
$$

where $A$ is given by Eq. (2.9). The eigenstates have the simple form

$$
\left|\lambda>=O_{\lambda}^{\dagger}\right|>=\sum_{p h} X_{p h}(\lambda) a_{p}^{\dagger} a_{h} \mid>.
$$


TDA is exact. It is nothing but the shell model of the nuclear Hamiltonian within the $p h$ space. It is, however, inconsistent with the HO-like Eqs. (2.4) which, strictly, hold only if $\mid 0>$ is the true, correlated, ground state. Just because these correlations are neglected, TDA is less general than RPA and less suitable for investigating collective modes.

While accounting for the Landau damping, neither TDA or RPA can describe the fragmentation induced by the coupling of the mode with complex configurations. In order to account for such a collisional damping, one has to go beyond mean-field and enlarge the space so as to allow for multiphonon excitations. This is achieved, for instance, in the QPM developed by Soloviev [24]. In this approach, a Hamiltonian of generalized separable form is expressed in terms of QRPA phonon operators and then diagonalized in a severely truncated space which includes a selected set of two and three RPA phonons. The method has been extensively and successfully adopted to describe both low and high energy multiphonon excitations in spherical as well as deformed nuclei. It has greatly contributed to clarify the microscopic structure of the mixed-symmetry states [25] and of the double giant dipole resonance [26]. The method has also been crucial in the understanding of the nature of the $0^{+}$states observed in large abundance in deformed nuclei [27].

Though very successful, the QPM has some limitations. It is suitable only for a Hamiltonian of separable form and is based on the quasi-boson approximation. One consequence of such an approximation is that the correlations in the ground state are accounted for only virtually.

Very recently, we have developed a new multiphonon approach which is free of the QBA and accounts explicitly for the correlations in the ground state [30]. The method is in principle exact and completely equivalent to shell model.

\section{A new multiphonon approach}

The goal of this new method is to generate a basis composed of multiphonon states $|n ; \alpha\rangle$, where $n=0,1,2, \ldots, N$ denotes the number of phonons constructed in TDA. The most obvious basis would be

$$
|n ; \alpha\rangle=\left|v_{1}, v_{2}, \ldots, v_{N}>=O_{v_{1}}^{\dagger} O_{v_{2}}^{\dagger} \ldots O_{v_{N}}^{\dagger}\right|>,
$$

where $\left|v_{i}\right\rangle$ is for instance the TDA phonon given by Eq. (2.18). A direct diagonalization in such a basis is simply prohibitive. We have therefore to find ways of circumventing the direct diagonalization.

Such a way out is suggested by the structure of the TDA state itself. Let us suppose in fact that the $(n-1)$-phonon states are known. We can then adopt for the $n$-phonon subspace a basis composed of the states

$$
b_{p h}^{\dagger} \mid n-1 ; \alpha>
$$

where $b_{p h}^{\dagger}=a_{p}^{\dagger} a_{h}$. For $n=1,|n-1 ; \alpha>=|>$ is simply the $p h$ vacuum and $b_{p h}^{\dagger} \mid>$ is just the TDA basis. For more than one phonon $n>1$, we have to keep in mind that our goal is to find, starting from the basis (3.2), the states $\mid n, \alpha>$ which bring the Hamiltonian $H$ to diagonal form within the $n$-phonon subspace. Having in mind this goal, and exploiting the fact that the $p h$ operators $a_{p}^{\dagger} a_{h}$ couple only subspaces which differ at most by one TDA phonon, we derive the following equations of motion

$$
<n ; \beta\left|\left[H, b_{p h}^{\dagger}\right]\right| n-1 ; \alpha>=\left(E_{\beta}^{(n)}-E_{\alpha}^{(n-1)}\right)<n ; \beta\left|b_{p h}^{\dagger}\right| n-1 ; \alpha>.
$$


We then write the Hamiltonian in second quantized form and expand the commutator $\left[H, a_{p}^{\dagger} a_{h}\right]$ on the left-hand side of the equation. After a linearization procedure, we obtain for the $n$-phonon subspace the eigenvalue equation

$$
\sum_{\gamma p^{\prime} h^{\prime}} A_{\alpha \gamma}^{(n)}\left(p h ; p^{\prime} h^{\prime}\right) X_{\gamma \beta}^{(n)}\left(p^{\prime} h^{\prime}\right)=E_{\beta}^{(n)} X_{\alpha \beta}^{(n)}(p h),
$$

where

$$
X_{\alpha \beta}^{(n)}(p h) \equiv<n ; \beta\left|b_{p h}^{\dagger}\right| n-1 ; \alpha>
$$

are the vector amplitudes and

$$
\begin{aligned}
A_{\alpha \gamma}^{(n)}\left(p h ; p^{\prime} h^{\prime}\right) & =\delta_{h h^{\prime}} \delta_{p p^{\prime}} \delta_{\alpha \gamma}^{(n-1)}\left[\left(\varepsilon_{p}-\varepsilon_{h}\right)+E_{\alpha}^{(n-1)}\right] \\
& +\sum_{h_{1}} V_{p^{\prime} h_{1} h^{\prime} p} \rho_{\alpha \gamma}^{(n-1)}\left(h_{1} h\right)-\sum_{p_{1}} V_{p^{\prime} h h^{\prime} p_{1}} \rho_{\alpha \gamma}^{(n-1)}\left(p p_{1}\right) \\
& +\frac{1}{2} \delta_{h h^{\prime}} \sum_{p_{1} p_{2}} V_{p^{\prime} p_{1} p p_{2}} \rho_{\alpha \gamma}^{(n-1)}\left(p_{1} p_{2}\right)-\frac{1}{2} \delta_{p p^{\prime}} \sum_{h_{1} h_{2}} V_{h h_{1} h^{\prime} h_{2}}\left\{\rho_{\alpha \gamma}^{(n-1)}\left(h_{1} h_{2}\right)\right\} .
\end{aligned}
$$

The quantity

$$
\rho_{\alpha \gamma}^{(n)}(k l)=<n ; \gamma\left|a_{k}^{\dagger} a_{l}\right| n ; \alpha>
$$

is the density matrix and plays a crucial role. It is, in fact, seen to weight the particle-hole, particleparticle and hole-hole interaction.

The simple structure of the equations is to be stressed. It is also to be pointed out that the above matrix $A^{(n)}$ contains only density matrices defined within the $(n-1)$-phonon subspace. For them, as we shall see, recursive relations hold which allow an iterative solution of Eq. (3.4). We can indeed start from the $p h$ vacuum $|n=0\rangle=\mid>$ and solve the equations step by step up to a given number of phonons. In the first step, $n=1$, since the $(n-1)$-phonon subspace contains only the $p h$ vacuum |\rangle , the density matrices appearing in Eq. (3.6) assume the values

$$
\rho^{(0)}\left(p p^{\prime}\right)=<\left|a_{p}^{\dagger} a_{p^{\prime}}\right|>=0, \quad \rho^{(0)}\left(h h^{\prime}\right)=<\left|a_{h}^{\dagger} a_{h^{\prime}}\right|>=\delta_{h h^{\prime}},
$$

so that the matrix $A^{(n=1)}$ becomes just the TDA Hamiltonian matrix (2.9). Thus, our equations (3.4) represent the most natural extension of the Tamm-Dancoff equations to multiphonon spaces. Their eigenvalues are relative energies with respect to the energy of the $p h$ vacuum.

The iterative process, however, cannot be implemented in the way we just did. In fact, when we move to subspaces with $n>1$, we run into the problem of the redundancy of the basis states.

\subsection{Generation of a redundancy-free multiphonon basis}

The basis states we have adopted are of the factorized form $b_{p h}^{\dagger}\left|n-1 ; \alpha>=\left(a_{p}^{\dagger} a_{h}\right)\right| n-1 ; \alpha>$, where the $p h$ operator $a_{p}^{\dagger} a_{h}$ acts on each phonon state $\mid n-1 ; \alpha>$ as a whole and not on its Fermion constituents. It is therefore impossible to enforce the Pauli principle. In the absence of such a constraint, the number of $p h$ states is determined independently of the phonon states $|n-1 ; \alpha\rangle$ and vice versa.

It follows that the states $b_{p h}^{\dagger} \mid n-1 ; \alpha>$ form an overcomplete set of linearly dependent states, yielding a redundant number of eigensolutions with spurious admixtures in each eigenvector. 
In order to eliminate this redundancy problem and obtain the correct number of spuriosity free eigenstates, we have to formulate an eigenvalue problem of general type. Let us denote by $N_{r}$ the total number of states $b_{p h}^{\dagger} \mid n-1 ; \alpha>$. It is always possible to expand the state to be determined $\mid n ; \beta>$ in terms of these redundant $N_{r}$ states

$$
\left|n ; \beta>=\sum_{\alpha p h} C_{\alpha \beta}^{(n)}(p h) b_{p h}^{\dagger}\right| n-1 ; \alpha>.
$$

Upon insertion into Eqs. (3.5) and (3.6), we get

$$
\begin{array}{r}
X=\mathscr{D} C \\
A \mathscr{D C}=E \mathscr{D} C,
\end{array}
$$

where $\mathscr{D}$ is the overlap or metric matrix

$$
d_{\alpha \beta}^{(n-1)}\left(p h ; p^{\prime} h^{\prime}\right)=\left\langle n-1 ; \beta\left|b_{p^{\prime} h^{\prime}} b_{p h}^{\dagger}\right| n-1 ; \alpha\right\rangle .
$$

Eq. (3.10) defines an eigenvalue equation of general form. It is, however, ill-defined. The matrix $\mathscr{D}$ is singular. Its determinant is necessarily vanishing, since the vectors $b_{p h}^{\dagger}|n-1 ; \alpha\rangle$ are not linearly independent.

The traditional prescriptions [31] adopted to overcome this problem are based on the straightforward diagonalization of $\mathscr{D}$, which is time consuming. We have avoided the direct diagonalization of $\mathscr{D}$ by adopting an alternative method based on the Choleski decomposition[30]. This provides a fast and efficient prescription for extracting a basis of linear independent states $b_{p h}^{\dagger} \mid n-1 ; \alpha>$ spanning the physical subspace of the correct dimensions $N_{n}<N_{r}$.

We are now ready to solve the generalized eigenvalue Eqs. (3.10). To this purpose we need to compute the matrices $A$ and $\mathscr{D}$. The calculation of the metric matrix $\mathscr{D}$ is a highly non trivial task. Elaborated diagrammatic techniques and complex iterative procedures have been envisaged to this purpose[28, 29, 32, 33].

Our equation of motion method provides the easiest and most natural solution to this latter problem by yielding the simple formula

$$
d_{\alpha \beta}^{(n-1)}\left(p h ; p^{\prime} h^{\prime}\right)=\sum_{\gamma}\left[\delta_{p p^{\prime}} \delta_{\gamma \beta}-\rho_{\gamma \beta}^{(n-1)}\left(p p^{\prime}\right)\right] \rho_{\alpha \gamma}^{(n-1)}\left(h h^{\prime}\right),
$$

where the matrix densities are computed by using the recursive relations

$$
\begin{aligned}
\rho_{\alpha \beta}^{(n)}\left(p_{1} p_{2}\right) & =\sum_{p h \gamma \delta} C_{\alpha \gamma}^{(n)}(p h) X_{\delta \beta}^{(n)}\left(p_{1} h\right)\left[\delta_{p p_{2}} \delta_{\gamma \delta}-\rho_{\gamma \delta}^{(n-1)}\left(p p_{2}\right)\right], \\
\rho_{\alpha \beta}^{(n)}\left(h_{1} h_{2}\right) & =\sum_{p h \gamma \delta} C_{\alpha \gamma}^{(n)}(p h)\left[\delta_{h h_{1}} \delta_{\gamma \delta} X_{\gamma \beta}^{(n)}\left(p h_{2}\right)-X_{\delta \beta}^{(n)}(p h) \rho_{\gamma \delta}^{(n-1)}\left(h_{1} h_{2}\right)\right] .
\end{aligned}
$$

The Matrix $A$ is also easily computed by exploiting the above Eqs. (3.13) and (3.14).

We have thus accomplished all necessary steps for solving the generalized eigenvalue problem (3.10). These can be summarized as follows:

1. Assuming that the eigensolutions in the $(n-1)$-phonon subspace are known, construct the matrix $A^{(n)}$ through Eq. (3.6). 
2. Compute the metric matrix $\mathscr{D}$.

3. Use the Choleski decomposition method to extract a set of $N_{n}$ linearly independent states and construct the $N_{n} \times N_{n}$ non singular matrix $\mathscr{D}_{n}$.

4. Solve the generalized eigenvalue equation in the $N_{n}$-dimensional physical subspace to obtain the correct number of eigenvalues and the expansion coefficients $C_{\alpha \beta}^{(n)}(p h)$ of the corresponding eigenvectors.

5. Compute within the $n$-phonon subspace the amplitudes $X_{\alpha \beta}^{(n)}(p h)$ using Eqs. (3.9) and the density matrix $\rho_{\alpha \beta}^{(n)}(k l)$ using the recursive relations (3.13) and (3.14).

6. $X_{\alpha \beta}^{(n)}(p h)$ and $\rho_{\alpha \beta}^{(n)}(k l)$ are the new entries for the equations of motion in the $(n+1)$-phonon subspace.

The iterative process is clearly outlined. To implement it, we have just to start with the lowest trivial 0-phonon subspace, the $p h$ vacuum, and, then, solve the eigenvalue problem step by step within each $n$-phonon subspace following the prescriptions 1 to 6 .

Such a procedure yields a multiphonon basis which reduces the Hamiltonian to diagonal blocks, mutually coupled by off-diagonal terms

$$
H=\sum_{n \alpha} E_{\alpha}^{(n)}|n ; \alpha\rangle\left\langle n ; \alpha\left|+\sum_{n \alpha n^{\prime} \beta}\right| n^{\prime} ; \beta\right\rangle\left\langle n^{\prime} ; \beta|H| n ; \alpha\right\rangle\langle n ; \alpha|,
$$

where $n^{\prime}=n \pm 1, n \pm 2$, since only subspaces differing from each others by two-phonons, at most, are coupled. The off-diagonal pieces are given by recursive formulas and, therefore, easily computed.

It remains now only to diagonalize the Hamiltonian matrix to get the exact nuclear eigenvalues and eigenvectors. These are given by

$$
\left|\Psi_{v}\right\rangle=\sum_{n, \alpha_{n}} C_{\alpha_{n}}^{(v)}\left|n ; \alpha_{n}\right\rangle
$$

Iterating Eq. (3.8) for the states $\left|n ; \alpha_{n}\right\rangle$, for each $n$, we get

$$
\left|\Psi_{v}\right\rangle=\sum_{n=0, N} \sum_{v_{1} . . v_{n}} C_{v_{1}, v_{2}, \ldots ., v_{n}}^{(v)}\left|n ; v_{1}, v_{2}, \ldots . . v_{n}\right\rangle,
$$

where $v_{i}$ labels TDA states and the coefficients $C_{v_{1}, v_{2}, \ldots, v_{n}}^{(v)}$ are very involved sums of products of expansion coefficients. Clearly, the above states, including the ground state, are highly correlated. Using the wave functions (3.16), we can compute the transition amplitudes

$$
\mathscr{M}(\text { if })=\left\langle\Psi_{v_{f}}|\mathscr{M}| \Psi_{v_{i}}\right\rangle
$$

of the one-body operator

$$
\mathscr{M}=\sum_{k l} \mathscr{M}_{k l} a_{k}^{\dagger} a_{l}
$$

obtaining

$$
\mathscr{M}(i f)=\left\langle\Psi_{v_{f}}|\mathscr{M}| \Psi_{v_{i}}\right\rangle=\sum_{k l n_{i} n_{f} \alpha_{i} \beta_{f}} \mathscr{M}_{k l} C_{n_{i} \alpha_{i}}^{\left(v_{i}\right)} C_{n_{f} \beta_{f}}^{*\left(v_{f}\right)} \rho_{\alpha_{i} \beta_{f}}^{\left(n_{i} n_{f}\right)}(k l)
$$

These amplitudes involve the density matrix, which can be easily computed. Their composite structure reflects the fact that all correlations are accounted for explicitly and not virtually as in RPA. 


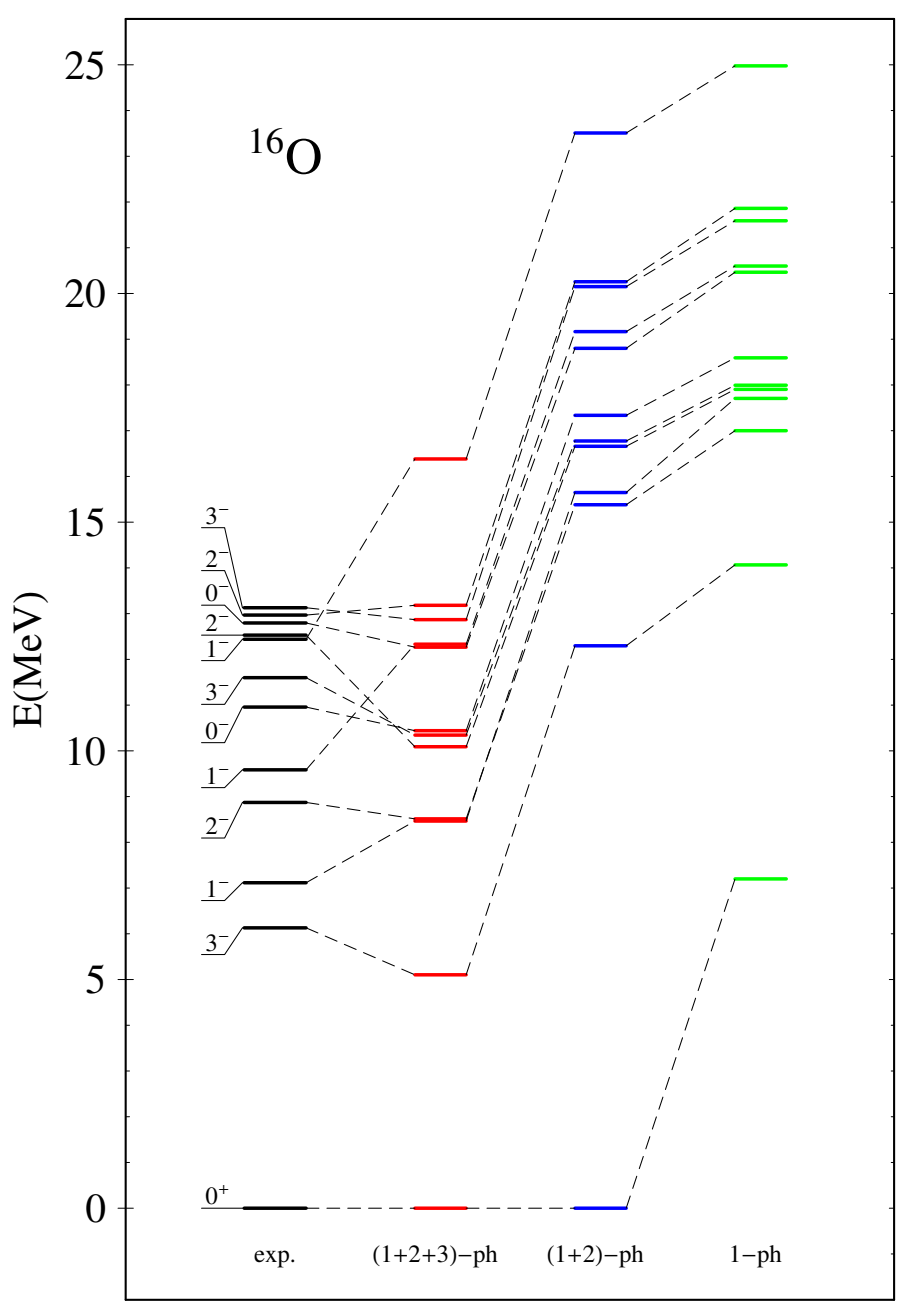

Figure 1: Low-lying negative parity spectrum in ${ }^{16} \mathrm{O}$. The energy of the one-phonon ground state, namely the $p h$ vacuum, is relative to the one of the correlated $0_{g s}^{+}$, assumed coincident with the experimental ground state level.

\section{A numerical illustrative application of the method for ${ }^{16} \mathrm{O}$}

For illustrative purposes, we apply the method to ${ }^{16} \mathrm{O}$, whose low-lying excitations are known to have a highly complex $p h$ structure since the pioneering work of Brown and Green [34].

The low-energy positive parity spectrum of this nucleus was studied in a shell model calculation which included up to $4 p-4 h$ and $4 \hbar \omega$ configurations [35]. The same spectrum was studied very recently within a no-core shell model and an algebraic symplectic shell model [36]. In both approaches, the model space was enlarged so as to include all configurations up to $6 \hbar \omega$.

We have included all $p h$ configurations up to $3 \hbar \omega$, which limits our phonon space up to $n=3$. 


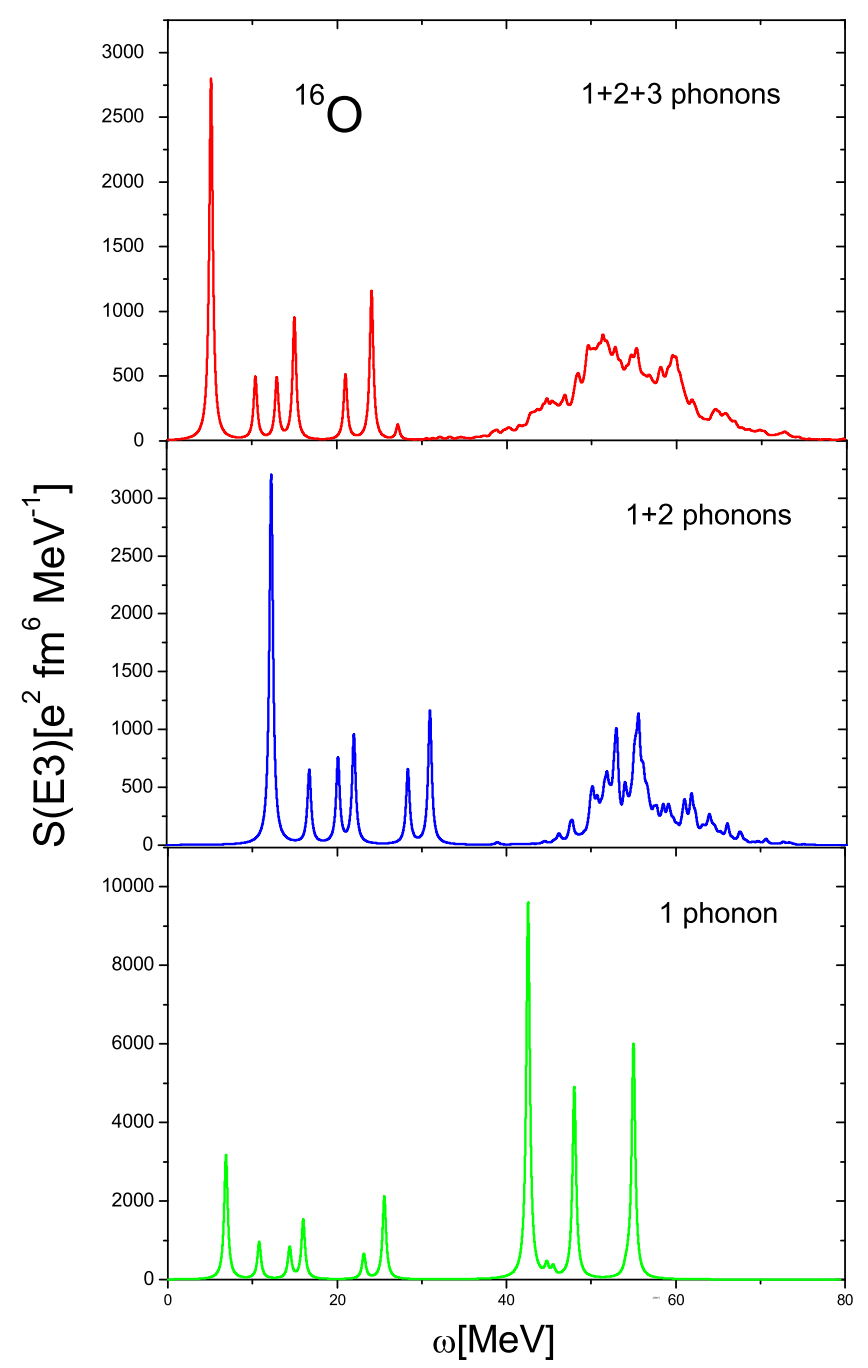

Figure 2: $E 3$ strength distributions in ${ }^{16} \mathrm{O}$

Such a space is considerably smaller than the one adopted in shell model. This is sufficient for our purpose, which is to show that our method can be implemented exactly. Moreover, our approach generates at once the whole spectrum of positive and negative parity states. This allows the study of low-lying as well as high energy spectroscopic properties and, in particular, the anharmonic features of the giant resonances. To this purpose, we used a modified harmonic oscillator one-body Nilsson Hamiltonian [37] plus a bare G-matrix deduced from the Bonn-A potential [38].

We have adopted the method of Palumbo [39] to separate the intrinsic from the center of mass motion. This method was applied to standard shell model by Glockner and Lawson [40] and, since then, widely adopted in nuclear structure studies. It consists of adding an HO Hamiltonian in the center of mass coordinates multiplied by a coupling constant. If all configurations up to $3 \hbar \omega$ are included, as in our case, each eigenfunction of the full Hamiltonian gets factorized into an intrinsic 
and center of mass components. For a large enough coupling constant, the center of mass excited states are pushed high up in energy, leaving at the low physical energies only the intrinsic states, namely the eigenfunctions with the center of mass in the ground state. Thus, a complete separation of the center of mass from the intrinsic motion is achieved.

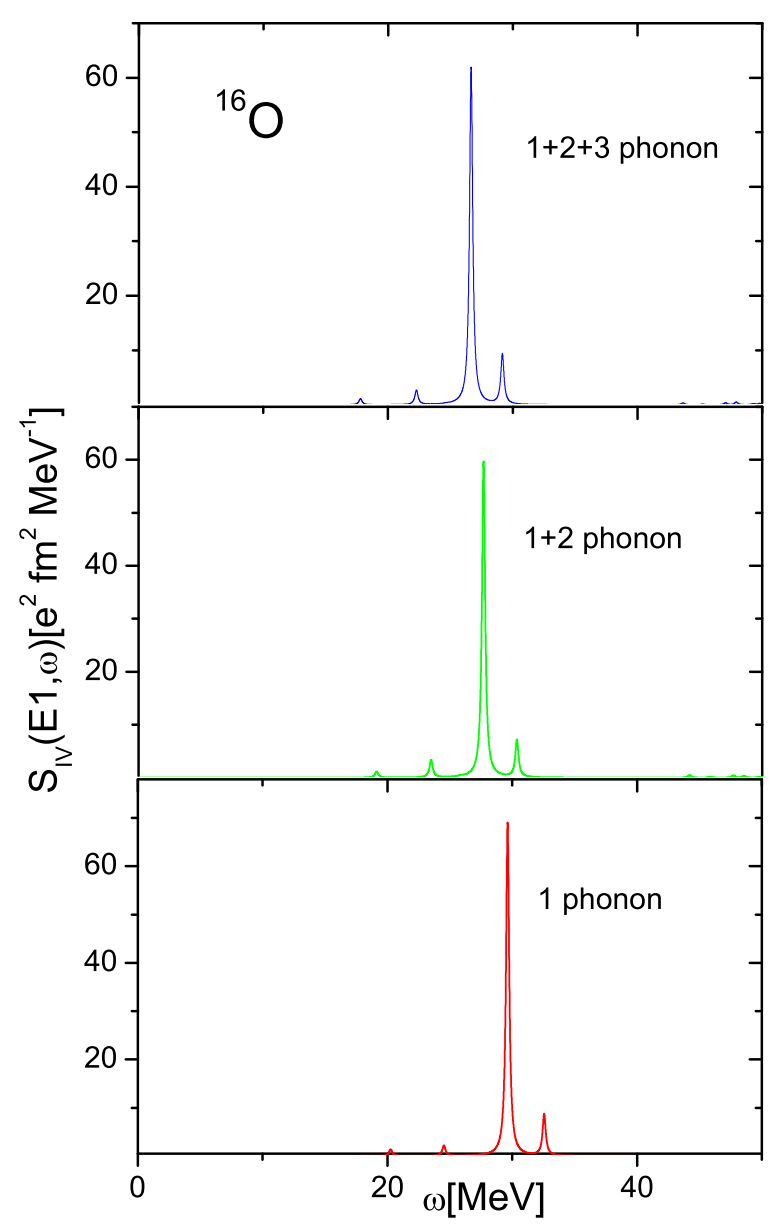

Figure 3: Isovector $E 1$ strength distributions in ${ }^{16} \mathrm{O}$

\subsection{Results}

Being the space confined to $3 \hbar \omega$, the ground state contains correlations up to 2-phonons only. These account for about $20 \%$ of the state, while the remaining $80 \%$ pertains to the ph vacuum. These values are reasonably close to the estimates of the consistent no-core symplectic shell model. This yielded about $60 \%$ for the $0 p-0 h, 20 \%$ for $2 p-2 h$ and $20 \%$ for the other more complex configurations[36], excluded from our restricted space.

As shown in Fig. 1, the effect of the multiphonon configurations on the low-lying negative parity spectrum is very important. The coupling with the two phonons pushes down the one-phonon 
levels. The energy shifts, however, are much smaller than the one induced by the positive parity two-phonons on the ground state, so that the gap between excited and ground state levels increases with respect to the one phonon case. The three phonon configurations are considerably more effective and bring all the states down in energy within the range of the corresponding experimental levels [41, 42, 43].

Let us now investigate the anharmonicities induced by the multiphonon configurations on some selected giant resonances. We have studied isoscalar and isovector dipole and quadrupole transitions. To this purpose, we have computed the strength function

$$
\mathscr{S}(\lambda, \omega)=\sum_{v} B_{v}(\lambda) \delta\left(\omega-\omega_{v}\right) \approx \sum_{v} B_{v}(\lambda) \rho_{\Delta}\left(\omega-\omega_{v}\right),
$$

where $\omega$ is the energy variable, $\omega_{v}$ the energy of the transition of multipolarity $\lambda$ from the ground to the $v_{t h}$ excited state $\Psi_{\lambda}^{(v)}$ of spin $J=\lambda$, given by Eq. (3.16), and

$$
\rho_{\Delta}\left(\omega-\omega_{v}\right)=\frac{\Delta}{2 \pi} \frac{1}{\left(\omega-\omega_{v}\right)^{2}+\left(\frac{\Delta}{2}\right)^{2}}
$$

is a Lorentzian of width $\Delta$, which replaces the $\delta$ function as a weight of the reduced transition probability [44]

$$
B^{(v)}(\lambda)=\sum_{\mu}\left|\left\langle\Psi_{\lambda \mu}^{(v)}|\mathscr{M}(\lambda \mu)| 0\right\rangle\right|^{2}
$$

For all electric $(E \lambda)$ transitions, we adopt the standard multipole operator

$$
\mathscr{M}(E \lambda \mu)=\frac{e}{2} \sum_{i=1}^{A}\left(1-\tau_{3}^{i}\right) r_{i}^{\lambda} Y_{\lambda \mu}\left(\hat{r}_{i}\right)
$$

where $\tau_{3}=1$ for neutrons and $\tau_{3}=-1$ for protons. The isoscalar $E 1$ transition deserves special attention. The $\tau=0$ component of the $\lambda=1$ operator (4.4) excites the center of mass spurious mode. It is remarkable that such a mode has been completely removed in our calculation. In order to excite the intrinsic isoscalar dipole mode, known as squeezed dipole mode, we have to go beyond the long wavelength approximation and use the operator

$$
\mathscr{M}_{I S}(\lambda=1 \mu)=\sum_{i=1}^{A} r_{i}^{3} Y_{1 \mu}\left(\hat{r}_{i}\right)
$$

It is important to notice the absence of the corrective term generally included in order to eliminate the spurious contribution due to the center of mass excitation. Such a term is not necessary in our approach which guarantees a complete separation of the center of mass from the intrinsic motion.

Fig. 2 shows the distribution of the $E 3$ strengths over a very large energy interval. The twophonon configurations have a damping and spreading effect and push the spectrum up in energy. The strength is further redistributed and shifted downward by the three phonon configurations.

It is interesting to investigate the phonon structure of some of these $3^{-}$states. While the low lying ones are mainly one-phonon states, those contributing to the main peaks at high energy are dominated by three phonon components.

The isovector $E 1$ response $S_{I V}(E 1, \omega)$ is only slightly affected by the multiphonon configurations (Fig. 3), an indication that the isovector giant dipole resonance mode is basically harmonic. 
More dramatic is the effect of the multiphonon excitations on the isoscalar $E 1$ response. The strength gets spread over a much larger energy range as we include these more complex configurations (Fig. 4). Such a spreading was expected. Indeed, the isoscalar giant dipole resonance is due to $p h$ excitations of $3 \hbar \omega$. This is also the energy of many $2 p-2 h$ as well as $3 p-3 h$ configurations which are therefore to be included in a consistent description of the mode.

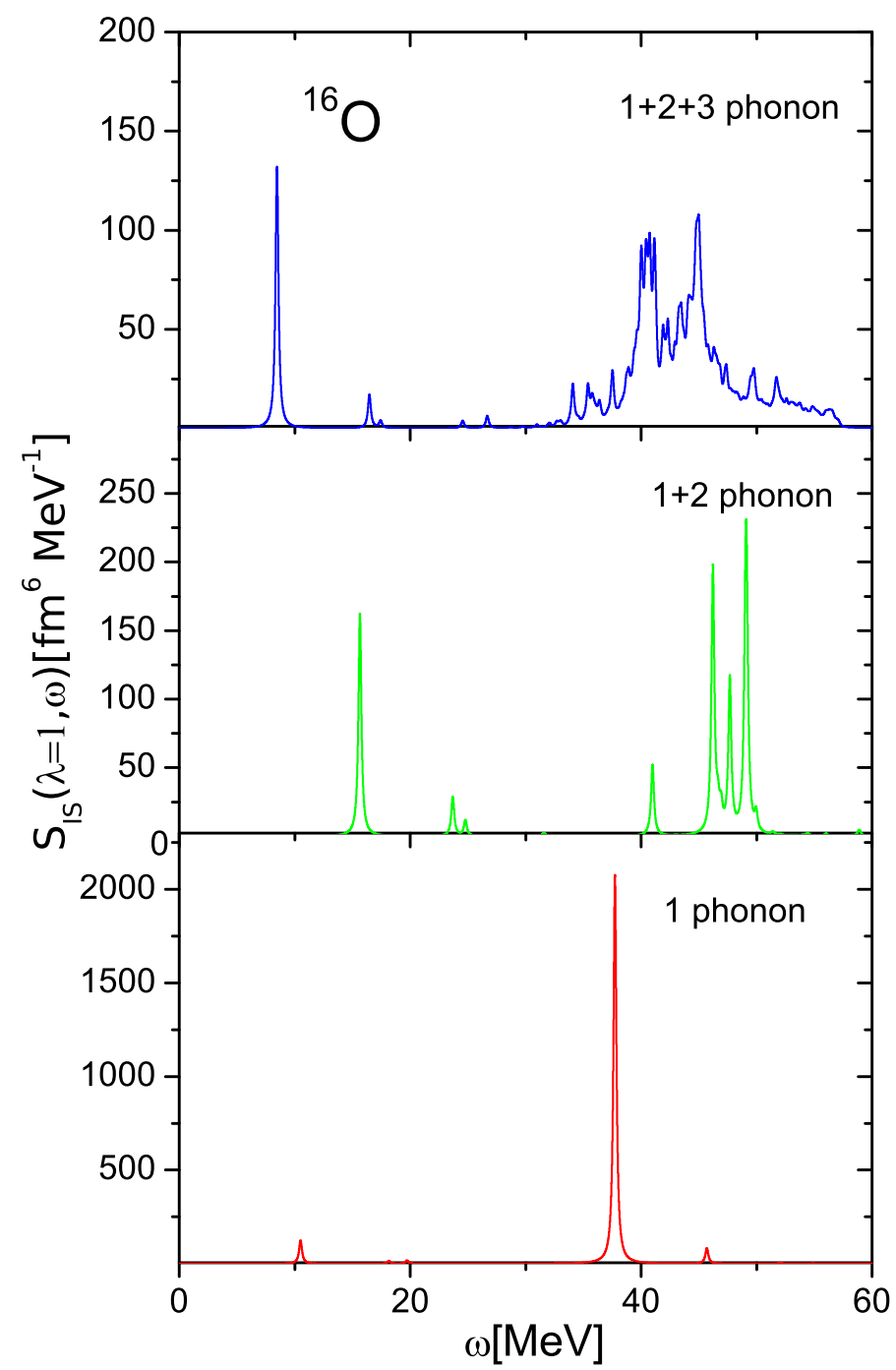

Figure 4: Isoscalar $E 1$ strength distributions in ${ }^{16} \mathrm{O}$

\section{Conclusions}

The method we have proposed yields eigenvalue equations of simple structure and of easy solution for a Hamiltonian of general form. The method is exact not only in principle but also in its actual numerical implementation, as shown here for ${ }^{16} \mathrm{O}$.

Indeed, no approximations of any sort have been made in the numerical treatment of such a nucleus. Such a calculation was confined to a space which included up to three-phonons and $3 \hbar \omega$, 
sufficient for our illustrative purposes, but too restricted to describe exhaustively and faithfully all spectroscopic properties of this complex nucleus.

In order to extend the method to spaces with a larger number of phonons we need to truncate the dimensions of the phonon subspaces. The highly correlated nature of the phonon basis generated by the eigenvalue equations suggests that such a truncation should be feasible with little detriment to the accuracy of the solutions. It is, in fact, conceivable that most TDA phonons are non collective and unnecessary. To this purpose we are developing a new formulation which should render the truncation procedure efficient and, at the same time, accurate. Such a new formulation should allow to select naturally the relevant TDA phonons and discard the others, thereby reducing drastically the number of TDA phonons coming into play. Such a severe cut should render possible the access to phonon subspaces with a quite large number $N$ of phonons and, thus, render the approach competitive with shell model even in describing the low energy spectroscopic properties. The method, however, goes beyond shell model. In fact, it allows to include in TDA phonons $p h$ configurations of arbitrarily high energy not at reach of shell model calculations.

The method can be extended in several ways. It can be reformulated so as to include RPA phonons. This extension, however, might be unnecessary since the method, already in its present TDA formulation, yields an explicitly correlated ground state.

A formulation of the method in terms of quasiparticle rather than particle-hole states is also straightforward and especially useful. It allows, indeed, to study anharmonicities and multiphonon excitations in open shell nuclei not easily accessible to shell model methods.

\section{References}

[1] D. J. Rowe, Nuclear Collective Motion (Methuen London, 1970).

[2] P. Ring and P. Schuck, The Nuclear Many/Body Problem (Springer-Verlag New York, 1980).

[3] P.F. Bortignon, A. Bracco, and R. A. Broglia, Giant Resonances (harwood academic publishers, Amsterdam,1998).

[4] M. Kneissl, H. H. Pitz and A. Zilges, Prog. Part. Nucl. Phys. 37 (1996) 439.

[5] N. Pietralla et al., Phys. Rev. Lett. 83 (1999) 1303.

[6] C. Fransen et al., Phys. Rev. C 71 (2005) 054304.

[7] M. Kneissl, N. Pietralla and A. Zilges, J. Phys. G: Nucl. Part. Phys. 32 (2006) R217.

[8] N. Frascaria, Nucl. Phys. A482 (1988) 245c.

[9] T. Auman, P. F. Bortignon, H. Hemling, Annu. Rev. Nucl. Part. Sci. 48 (1998) 351.

[10] M. Krticka et al. Phys. Rev. Lett. 92, 172501 (2004).

[11] N. Lo Iudice and F. Palumbo, Phys. Rev. Lett. 41, 1532 (1978).

[12] D. Bohle et al. Phys. Lett. B 137, 27 (1984).

[13] S. R. Lesher et al, Phys. Rev. C 66, 051305(R) (2002).

[14] H.-F. Wirth et al, Phys. Rev. C 69, 044310 (2004).

[15] D. Bucurescu et al, Phys. Rev. C 73, 064309 (2006). 
[16] D. A. Meyer et al., Phys. Rev. C 74, 044309 (2006).

[17] S. T. Belyaev and V. G. Zelevinsky, Nucl. Phys. 39 (1962) 582.

[18] T. Marumori, M. Yamamura, and A. Tokunaga, Progr. Thor. Phys. 31 (1964) 1009.

[19] For a review see A. Klein and E. R. Marshalek, Rev. Mod. Phys. 63 (1991) 375.

[20] For a review see A. Arima and F. Iachello, Adv. Nucl. Phys. 13 (1984) 139.

[21] T. Otsuka, A. Arima, and F. Iachello, Nucl. Phys. A309, 1 (1978).

[22] A. Bohr and B. R. Mottelson, Nuclear Structure Vol. II (Benjamin, New York, 1975) and references therein.

[23] P. F. Bortignon, R. A. Broglia, D. R. Bes, and R. Liotta, Phys. Rep. 30 (1977) 305 and references therein.

[24] V. G. Soloviev, Theory of Atomic Nuclei: Quasiparticles and Phonons, (Institute of Physics, Bristol, 1992).

[25] N. Lo Iudice and Ch. Stoyanov, Phys. Rev. C 62 (2000) 047302; Phys. Rev. C 65 (2002) 064304.

[26] V. Yu. Ponomarev, P. F. Bortignon, R. A. Broglia, and V. V. Voronov, Phys. Rev. Lett. (2000) 851400.

[27] N. Lo Iudice, A. V. Sushkov, and N. Yu. Shirikova, Phys. Rev. C 70, 064316 (2004); Phys. Rev. C 72, 034303 (2005).

[28] C. Pomar, J. Blomqvist, R. J. Liotta, and A. Insolia, Nucl. Phys. A515 (1990) 381.

[29] M. Grinberg, R. Piepenbring, K. V. Protasov, B. Silvestre-Brac, Nucl. Phys. A597 (1996) 355.

[30] F. Andreozzi, N. Lo Iudice, and A. Porrino, F. Knapp, J. Kvasil, Phys. Rev. C 75, 044312(2007).

[31] D. J. Rowe, J. Math. Phys. 10 (1969) 1774.

[32] R. J. Liotta and C. Pomar, Nucl. Phys. (1982) A382 1.

[33] K. V. Protasov, B. Silvestre-Brac, R. Piepenbring, and M. Grinberg, Phys. Rev. C 53 (1996) 1646.

[34] G. E. Brown, A. M. Green, Nucl. Phys. 75 (1966) 401.

[35] W. C. Haxton and C. J. Johnson, Phys. Rev. Lett. 65 (1990) 1325.

[36] T. Dytrych, K. D. Sviratcheva, C. Bahri, J. P. Draayer, J.P. Vary, Phys. Rev. Lett. 98, 162503 (2007).

[37] P. Ring and P. Schuck, The Nuclear Many-Body Problem (Springer-Verlag, New York, 1980).

[38] R. Machleidt, Adv. Nucl. Phys. 19 (1989) 189.

[39] F. Palumbo, Nuc. Phys. 99 (1967) 100.

[40] D. H. Glockner and R. D. Lawson, Phys. Lett. B 53 (1974) 313.

[41] D.R. Tilley, H.R. Weller and C.M. Cheves, Nucl. Phys. A564 1 (1993).

[42] D. L. Berman and S. C. Filtz, Rev. Mod. Phys. 47 (1975) 713.

[43] Y.-W. Lui, H. L. Clark, and D. H. Youngblood, Phys. Rev. C 64 (2001) 064308.

[44] See for instance J. Kvasil, N. Lo Iudice, V.O. Nesterenko, and M. Kopal, Phys. Rev. C 58(1998) 209. 